\title{
Exploiting a Probabilistic Hierarchical Model for Generation
}

\author{
Srinivas Bangalore and Owen Rambow \\ AT\&T Labs - Research \\ 180 Park Avenue \\ Florham Park, NJ 07932 \\ \{srini, rambow\}oresearch.att.com
}

\begin{abstract}
Previous stochastic approaches to generation do not include a tree-based representation of syntax. While this may be adequate or even advantagcous for some applications, other applications profit from using as much syntactic knowledge as is available, leaving to a stochastic model only those issues that are not determined by the grammar. We present initial results showing that a trec-based model derived from a trec-annotated corpus improves on a tree model derived from an unannotated corpus, and that a trec-based stochastic model with a handcrafted grammar outperforms both.
\end{abstract}

\section{Introduction}

For many applications in natural language gencration (NLG), the range of linguistic expressions that must be generated is quite restricted, and a grammar for generation can be fully specified by hand. Moreover, in many cases it is very important not to deviate from certain linguistic standards in generation, in which case handcrafted grammars give excellent control. However, in other applications for NLG the variety of the output is much bigger, and the demands on the quality of the output somewhat less stringent. A typical example is NLG in the context of (interlingua- or transfer-based) machine translation. Another reason for relaxing the quality of the output may be that not enough time is available to develop a full grammar for a new target language in NLG. In all these cases, stochastic ("empiricist") methods provide an alternative to hand-crafted ("rationalist") approaches to NLG. To our knowledge, the first to use stochastic techniques in NLG were Langkilde and Knight (1998a) and (1998b). In this paper, we present Fergus (Flexible Empiricist/Rationalist Generation Using Syntax).
FERGUs follows Langkilde and Knight's seminal work in using an n-gram language model, but wo augment it with a trec-based stochastic model and a traditional tree-based syntactic grammar. More recent work on aspects of stochastic gencration include (Langkilde and Knight, 2000), (Malouf, 1999) and (Ratnaparkhi, 2000).

Before we describe in more detail how we use stochastic models in NJG, we recall the basic tasks in NLG (Rambow and Korclsky, 1992; Reiter, 1994). During text planning, content and structure of the target text are determined to achieve the overall communicative goal. During sentence planning, linguistic means - in particular, lexical and syntactic means - are determined to convey smaller pieces of meaning. During realization, the specification chosen in sentence planning is transformed into a surface string, by linearizing and inflecting words in tho sentence (and typically, adding function words). As in the work by Langkilde and Knight, our work ignores the text planning stage, but it does address the sentence planning and the realization stages.

The structure of the paper is as follows. In Section 2, we present the underlying grammatical formalism, lexicalized tree-adjoining grammar (LTAG). In Section 3, we describe the architecture of the system, and some of the modules. In Section 4 we discuss three experiments. In Section 5 we compare our work to that of Langkilde and Knight (1998a). We conclude with a summary of on-going work.

\section{Modeling Syntax}

In order to model syntax, we use an existing wide-coverage grammar of English, the XTAG grammar developed at the University of Pennsylvania (XTAG-Group, 1999). XTAG is a trecadjoining grammar (TAG) (Joshi, 1987a). In 


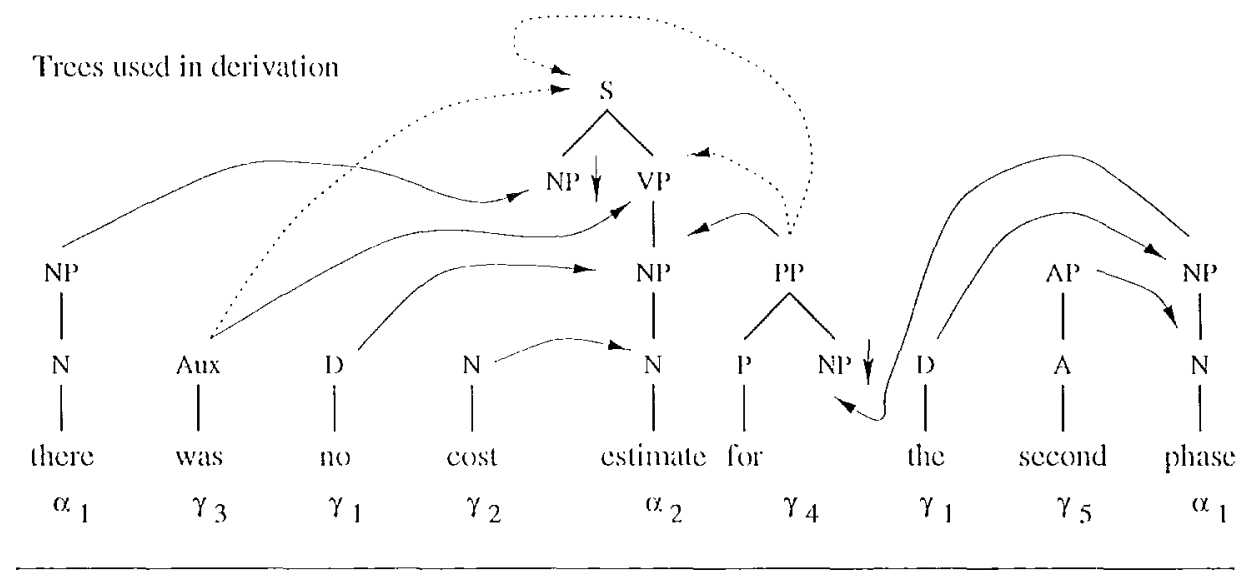

Other supertags for the lexemes found in the training corpus:

\begin{tabular}{|c|c|c|c|c|c|c|c|}
\hline none & $\alpha_{4}$ & $\alpha_{1}$ & $x_{1}$ & $\alpha_{1}$ & $\alpha_{4}$ & none & $\alpha_{3}$ \\
\hline & $a_{5}$ & & $*_{2}$ & $\gamma_{2}$ & $\alpha_{5}$ & & $x_{1}$ \\
\hline
\end{tabular}

Figure 1: An exeerpt from the XTAG grammar to derive There wes no cost estimate for the second phase; dotted lines show possible adjunctions that were not made

a TAG, the elementiary structures are phrasestructure trees which are composed using two operations, substitution (which appends one tree at the frontier of another) and adjunction (which inserts one tree into the minddle of another). In graphical representation, nodes at which substitution can take place are marked with down-arrows. In linguistic uses of TAG, we associate one lexical iten (its anchorr) with ach tree, and one or (typically) more trees with ach lexical item; as a result we obtain a lexicalized TAG or LTAG. Since oach lexical item is associated with a whole tree (rather than just a phrase-structure rule, for eximnple), we can specify both the predicatio-argument; structure of the lexeme (by including nodes at which its arguments must substitute) and morphosyntactic constraints such as subject-verb agreement within the structure associated with the lexeme. This property is referred to as TAG'S extended domain of locality. Note that in an LTAG, there is no distinction between lexicon and grammar. A sample grammar is shown in Figure 1.

We depart from XTAG in our treatment of trees for adjuncts (such as adverbs), and instead follow McDonald and Pustcjovsky (1985). While in XTAG the elementary tree for an adjunct contains phrase structure that attaches the adjunct to nodes in another tree with the

\begin{tabular}{|c|c|l|c|}
\hline stag & anchored by & adjoins to & direction \\
\hline$\gamma_{1}$ & Jet & NI' & right \\
$\gamma_{2}$ & $\mathrm{~N}$ & $\mathrm{~N}$ & right \\
$\gamma_{3}$ & $\Lambda \mathrm{ux}$ & $\mathrm{S}, \mathrm{VP}$ & right \\
$\gamma_{1}$ & Prep & $\mathrm{NP}, \mathrm{Vl}^{\prime}$ & left \\
& $o r$ & $\mathrm{~S}$ & right \\
$\gamma_{5}$ & Adj & $\mathrm{N}$ & right \\
\hline
\end{tabular}

Figure 2: Adjunction table for grammar fragment

specified label (say, VP) from the specified direction (say, from the left), in our system the trees for adjuncts simply express their active valency, but not how they comnect to the lexical item they modify. This information is kept, in the adjunction table which is associated with the granmar; an excerpt is shown in Figure 2. Trees that can adjoin to other trees (and have entries in the adjunction table) are called gamma-trees, the other trees (which can only be substituted into other trees) are alpha-trees.

Note that we can refer to a tree by a combination of its name, called its supertag, and its anchor. For: example, $\alpha_{1}$ is the supertag of an alpha-tree anchored by a noun that projects up to N], while $\gamma_{2}$ is the supertag of a gamma tree anchored by a nom that only projects to $\mathrm{N}$ (we 
assume adjectives are adjoined at $N$ ), and, as the adjunction table shows, can right-adjoin to an N. So that estimate $\alpha_{2}$ is a particular tree in our LTAG grammar. Another tree that a supertag can be associated with is $\alpha_{2}$, which represents the predicative use of a noun. ${ }^{1}$ Not all nouns are associated with all nominal supertags: the expletive there is only an $\alpha_{1}$.

When we derive a sentence using an LTAG, we combine elementary trees from the grammar using adjunction and substitution. For example, to derive the sentence There was no cost estimate for the second phase from the grammar in Figure 1, we substitute the trce for there into the tree for estimate. We then adjoin in the trees for the auxiliary was, the determiner no, and the modifying noun cost. Note that these adjunctions occur at different nodes: at VP, NP, and N, respectively. Wo then adjoin in the preposition, into which we substitute phase, into which we adjoin the and second. Note that all adjunctions are by gamma trces, and all substitution by alpha trees.

If we want to represent this derivation graphically, we can do so in a derivation tree, which we obtain as follows: whenever we adjoin or substitute a tree $t_{1}$ into a tree $t_{2}$, we add a now daughter labeled $t_{1}$ to the node labeled $t_{2}$. As explained above, the name of each tree used is the lexeme along with the supertag. (We omit the address at which substitution or adjunction takes place.) The derivation tree for our derivation is shown in Figure 3. As can be seen, this structure is a dependency tree and resembles a representation of lexical argument structure.

Joshi (1987b) claims that TAG's properties make it particularly suited as a syntactic representation for generation. Specifically, its extended domain of locality is useful in gencration for localizing syntactic properties (including word order as well as agreement and other morphological processes), and lexicalization is useful for providing an interface from semantics (the derivation tree represent the sentence's predicate-argument structure). Indeed, LTAG has been used extensively in generation, starting with (McDonald and Pustejovsky, 1985).

\footnotetext{
${ }^{1}$ Sentences such as Peter is a doctor can be analyzed with with be as the head, as is more ustual, or with doctor as the head, as is done in XTAG becanse the be rcally behaves like an auxiliary, not like a full verb.
}

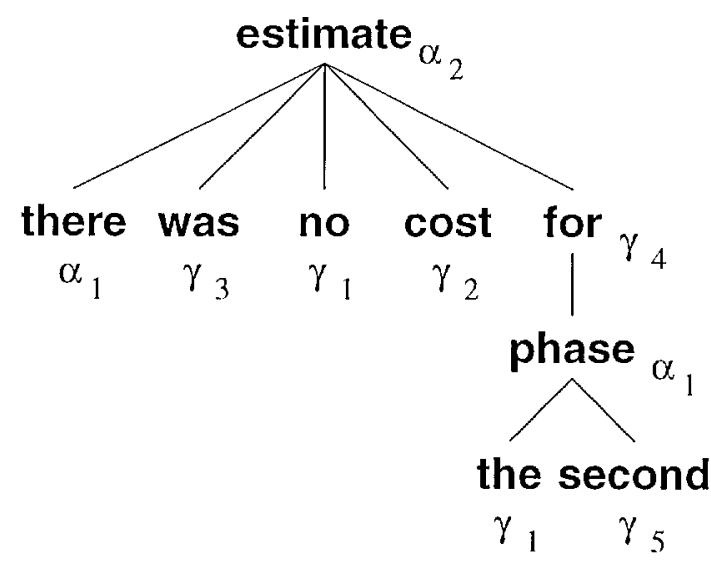

Figure 3: Derivation trec for LTAG derivation of There was no cost estimate for the second phase

\section{System Overview}

Fengus is composed of three modules: the Tree Chooser, the Unraveler, and the Linear Precedence (LP) Chooser. The input to the system is a dependency tree as shown in Figure 4. Note that the nodes are labeled only with lexemes, not with supertags. ${ }^{2}$ The Thee Chooser then uses a stochastic tree model to choose TAG trees for the nodes in the input structure. This step can be seen as analogous to "supertagging" (Bangalore and Joshi, 1999), except that now supertags (i.e., names of trees) must be found for words in a tree rather than for words in a linear sequence. The Unraveler then uses the XTAG grammar to produce a lattice of all possible linearizations that are compatible with the supertagged tree and the XTAG. The LP Chooser then chooses the most likely traversal of this lattice, given a language model. Wo discuss the three components in more detail.

The Tree Chooser draws on a tree model, which is a representation of XTAG derivation for 1,000,000 words of the Wall Street Journal. ${ }^{3}$ The Tree Chooser makes the simplifying as-

\footnotetext{
${ }^{2}$ In the system that we used in the experiments described in Section 4, all words (including function words) need to be present in the input representation, fully inflected. This is of course unrealistic for applications. In this paper, we only aim to show that the use of a Tree Model improves performance of a stochastic generator. See Section 6 for further discussion.

${ }^{3}$ This was constructed from the Penn Tree Bank using some heuristics, since the Penn Tree Bank does not contain full head-dependent information; as a result of the use of heuristics, the Tree Model is not fully correct.
} 


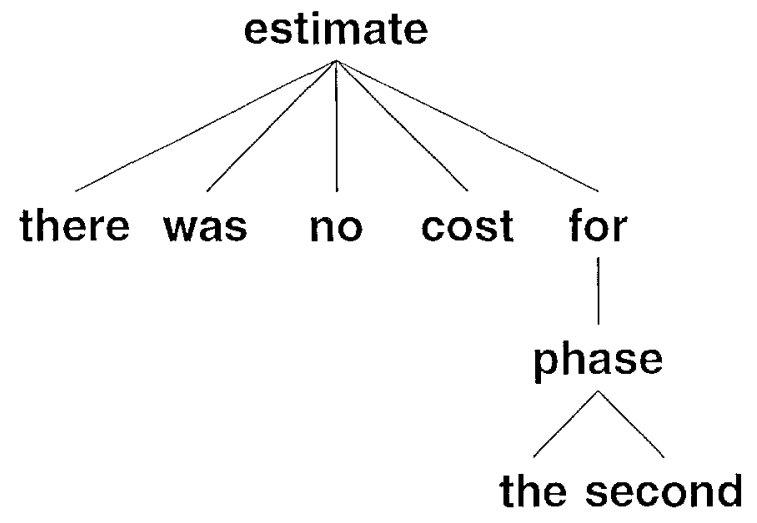

Figure 4: Input; to Fengus

sumptions that the choice of a tree for a node? depends only on its danghter nodes, thus allowing for a top-down dynamic programming algorithm. Specifically, a node $\eta$ in the input; structure is assigned a supertag s so that the probat bility of finding the treclet composed of $\eta$ with supertag $s$ and all of its danghters (als found in the input structure) is maximized, and such that $s$ is compatible with $\eta$ 's mother and her supertag $s_{m}$. Here, "compatible" means that the tree represented by $s$ can be adjoined or substituted into the trec represented by $s_{m}$, according to the XTAG grammar. For our example sentence, the input to the system is the tree shown in ligures 4, and the output from the Tree Chooser is the tree ats shown in ligure 3 . Note that while a derivation taee in 'IAG fully speceifies a derivation and thens a surface scontence, the output from the Tree Chooser does not. There are two reasons. Firstly, as explained at the ond of Section 2, for us trees corresponding to adjuncts are underspecified with respect to the adjunction site and/or the adjunction direction (from left or from sightit) in the tree of the mother node, or they may be mordered with respect to other adjuncts (for examp)le, the famous adjective ordering problem). Secondly, supertiags may have been chosen incorrectly or not at all.

The Unraveler takes as input the semispecified derivation tree (Figure 3) and produces a word lattice. Fach node in the derivation tree consists of a lexical item and a supertag. The linear order of the daughters with respect to the hear position of a supertag is specified in the XTAG grammar. This information is consulted to order the daughter nodes

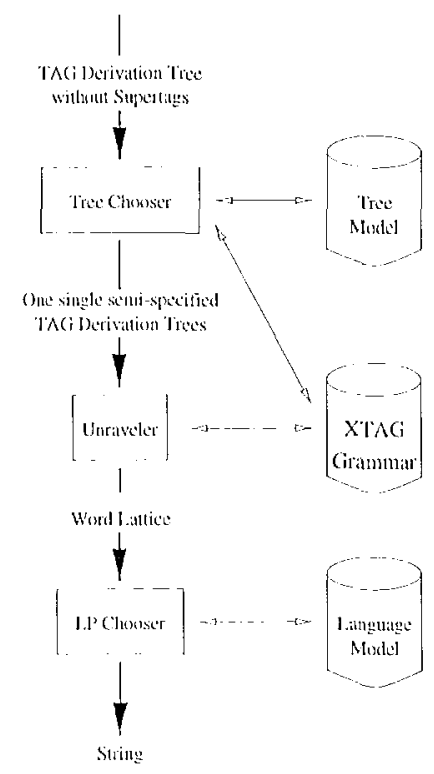

ligure 5: Architecture of Firgus

with respect to the head at oach level of the derivation tree. In cases where a daughter node (an be atticked at more than one place in the head supertag (as is the case in our example for was and for), a disjunction of all these positions are assigned to the daughter node. A bottomup algorithun then constructs a lattice that encodes the strings represented by each level of the derivation tree. The latitice at the reot of the derivation tree is the result of the Unraveler. The resulting latities for the example sentence is shown in lighere 6.

'The lattice ontputi from the Unraveler encodes all possible, word sequences permitited by the derivation structure. We rank these word sequences in the order of their likelihood by composing the lattice with a finitestate machine rejpresenting a trigram language model. 'This model has been constructed from $1,000,0000$ words of Wall Strect Jomrnal corpus. Wo pick the best path through the lattice resulting from the composition using the Viterloi algorithm, and this top ranking word sequence is the output of the LP' Chooser.

\section{Experiments and Results}

In order to show that the use of a tree model and a grammar does indeed help performance, we performed three experiments: 


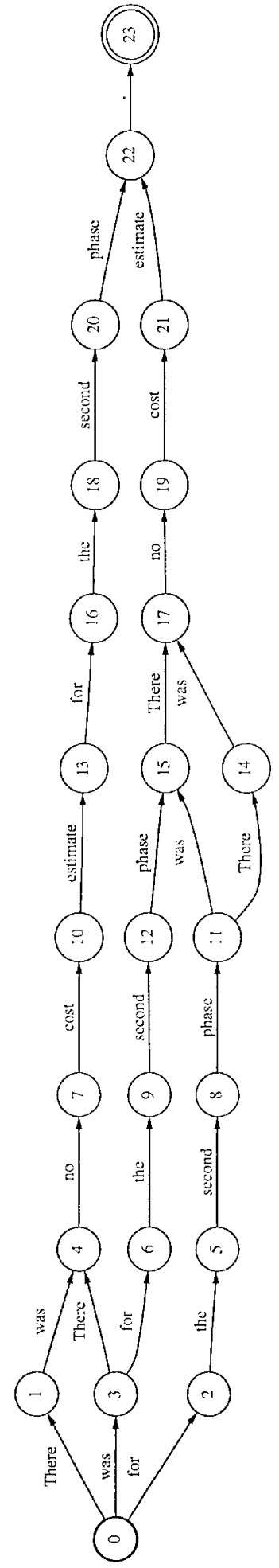

Figure 6: Word lattice for example sentence after Tree Chooser and Unraveler using the supertag-based model
- For the bascline expcriment, we impose a random tree structure for each sentence of the corpus and build a Tree Model whose parameters consist of whether a lexeme $l_{d}$ precedes or follows her mother lexeme $l_{m}$. We call this the Baseline Left-Right (LR) Model. This model generates There was estimate for phase the second no cost. for our example input.

- In the second experiment, we derive the parameters for the JJR model from an annotated corpus, in particular, the XTAG derivation tree corpus. This model generates There no estimate for the second phase was cost . for our example input.

- In the third experiment, as described in Section 3, we employ the supertag-based tree model whose parameters consist of whether a lexeme $l_{d}$ with supertag $s_{d}$ is a dependent of $l_{m}$ with supertag $s_{m}$. Furthermore we use the supertag information provided by the XTAG grammar to order the dependents. This model generates There was no cost estimate for the second phase . for our example input, which is indeed the sentence found in the WSJ.

As in the case of machine translation, cvaluation in generation is a complex issue. We use two metrics suggested in the MT literature (Alshawi et al., 1998) based on string edit, distance between the output of the generation system and the reference corpus string from the WSJ. These metrics, simple accuracy and generation accuracy, allow us to evaluate without human intervention, automatically and objectively. ${ }^{4}$

Simple accuracy is the number of insertion $(I)$, delction $(D)$ and substitutions $(S)$ errors between the target language strings in the test corpus and the strings produced by the generation model. The metric is summarized in Equation (1). $R$ is the number of tokens in the target string. This metric is similar to the string distance metric used for measuring speech recognition accuracy.

$$
\text { SimpleAccuracy }=\left(1-\frac{I+D+S}{R}\right)
$$

\footnotetext{
${ }^{4}$ We do not address the issue of whether these metrics can be used for comparative evaluation of other generation systems.
} 


\begin{tabular}{|c|c|c|c|}
\hline $\begin{array}{c}\text { Iree } \\
\text { Model }\end{array}$ & $\begin{array}{c}\text { Simple } \\
\text { Accuracy }\end{array}$ & $\begin{array}{c}\text { Generation } \\
\text { Accuracy }\end{array}$ & $\begin{array}{c}\text { Average time } \\
\text { per sentence }\end{array}$ \\
\hline Baseline LR Model & $41.2 \%$ & $56.2 \%$ & $186 \mathrm{~ms}$ \\
\hline Theebank derived LIR Model & $52.9 \%$ & $66.8 \%$ & $129 \mathrm{~ms}$ \\
\hline Supertag-based Model & $58.9 \%$ & $72.4 \%$ & $517 \mathrm{~ms}$ \\
\hline
\end{tabular}

Table 1: Performance results firom the three tree models.

Unlike speech recognition, the task of generation involves reordering of tokens. The simple accuracy metric, however, penalizes a misplaced token twice, as a deletion from its expected position and insertion at a different position. Wo use a second metric, Generation $\Lambda$ cenuacy, slown in Equation (2), which treats delotion of a token at one location in the string and the insertion of the same token at another location in the string as one single movenent exor (M). This is in addition to the remaining insertions $\left(I^{\prime}\right)$ and deletions $\left(D^{\prime}\right)$.

Generation Accuraciy $=\left(1-\frac{M+I^{\prime}+I^{\prime}+S}{R}\right)$

The simple accuracy, generation acemacy and the average time for generation of each test sentence for the three experinents are tabulated in Table 1. The test set consisted of 100 randomly chosen WSJ sentence with an average length of 16 words. As can be seen, the supertigg-based model improves over the LR model derived from amotated data and both models innprove over the baseline LR model.

Supertags incorporate richer information such as argument and adjunct distinction, and number and types of arguments. Wo expect to improve the performance of the supertag-based model by taking these features into account.

In ongoing work, we have developed trecbased metries in addition to the string-based presented here, in order to evaluate stochastic generation models. We have also attompted to corrolate these quantitative metries with human qualitative judgements. $\Lambda$ detailed discussion of these experiments and results is presented in (Bangalore et al., 2000).

\section{Comparison with Langkilde \& Knight}

Langkilde and Knight (1998a) use a handcrafted grammar that maps semantic representations to sequences of words with linearization constraints. A complex semantic structure is tamslated to a lattice, and a bigram language model then chooses among the possible surface strings encoded in the latitice.

'The system of Langkilde \& Knight, Nitrogen, is similar to Fergus in that generation is divided into two phases, the first of which results in a lattice from which a surface string is chosen during the second phase using a language nodel (in our case a trigram model, in Nitrogen's casse a bigrann model). However, the first phases are guite different. In fisirgus, we start with a lexical predicate-argument structure, while in $\mathrm{Ni}$ trogen, a more semantic inputi is used. Fragus conld casily be angmented with a preprocessor that maps a semantic representation to our syntactic input; this is not the focus of onr research. However, there are two more important differences. lirst, the hand-crafted grammar in Nitrogen maps directly from semantics to a linear representation, skipping the arborescent representation usually favored for the representation of syntax. There is no stochastic tree model, since there are no trees. In Fergus, initial choices are made stochastically based on the tree representation in the Tree Chooser. This allows us to capture stochastically certain longdistance effects which n-grams camnot, such as separation of parts of a collocations (such as perform an operation) through interposing adjuncts (John performed a long, somewhat tedious, and quite frustrating operation on his border collie). Second, the hand-crafted grammar used in FERGUS was crafted indepondently from the neod for generation and is a purcly declarative representation of English syntiax. $\Lambda$ s 
such, we can use it to handle morphological effects such as agrecment, which cannot in general be done by an n-gram model and which are, at the same time, descriptively straight forward and which are handled by all non-stochastic generation modules.

\section{Conclusion and Outlook}

We have presented empirical evidence that using a tree model in addition to a language model can improve stochastic NLG.

FerGus as presented in this paper is not ready to be used as a module in applications. Specifically, we will add a morphological component, a component that handles function words (auxiliaries, determiners), and a component that handles punctuation. In all threc cases, we will provide both knowledge-based and stochastic components, with the aim of comparing their behaviors, and using one type as a back-up for the other type. Finally, we will explore FERGUS when applied to a language for which a much more limited XTAG grammar is available (for example, specifying only the basic sentence word order as, say, SVO, and specifying subjectverb agrecment). In the long run, we intend Fergus to become a flexible system which will use hand-crafted knowledge as much as possible and stochastic models as much as necessary.

\section{References}

Hiyan Alshawi, Srinivas Bangalore, and Shona Douglas. 1998. Automatic accuisition of hicrarchical transduction models for machine tr anslation. In Proceedings of the 36th Annual Meeting Association for Computational Linguistics, Montreal, Canada.

Srinivas Bangalore and Aravind Joshi. 1999. Supertagging: An approach to almost parsing. Computational Linguistics, 25(2).

Srinivas Bangalore, Owen Rambow, and Steve Whittaker. 2000. Evaluation Metrics for Generation. In Proceedings of International Conference on Natural Language Generation, Mitzpe Ramon, Isreal.

Aravind K. Joshi. 1987a. An introduction to Tree Adjoining Grammars. In A. ManasterRamer, editor, Mathematics of Language, pages 87-115. John Benjamins, Amsterdam.

Aravind K. Joshi. 1987b. The relevance of tree adjoining grammar to gencration. In
Gerard Kempen, editor, Natural Language Generation: New Results in Artificial Intelligence, Psychology and Linguistics, pages 233-252. Kluwer Academic Publishers, Dordrecht/Boston/Lancaster.

Irene Langkilde and Kevin Knight. 1998a. Generation that exploits corpus-based statistical knowledge. In 36th Meeting of the Association for Computational Linguistics and 17th International Conference on Computational Linguistics (COLING-ACL'98), pages 704710, Montrćal, Canada.

Irene Langkilde and Kevin Knight. 1998b. The practical value of n-grams in generation. In Proceedings of the Ninth International Natural Language Generation Workshop (INLG'98), Niagara-on-the-Lakc, Ontario.

Irene Langkilde and Kevin Knight. 2000. Forest-based statistical sentence generation. In Proceedings of First North American ACL, Seatile, USA, May.

Robert Malouf. 1999. Two methods for predicting the order of prenominal adjectives in english. In Proceedings of CLIN99.

David D. McDonald and James D. Pustejovsky. 1985. Tags as a grammatical formalism for gencration. In 23rd Mecting of the Association for Computational Linguistics ( $A C L$ '85), pages 94-103, Chicago, IL.

Owen Rambow and Tanya Korelsky. 1992. $\Lambda_{\mathrm{p}}$ plied text gencration. In Third Conference on Applied Natural Language Processing, pages 40-47, Trento, Italy.

Adwait Ratnaparkhi. 2000. Trainable methods for surface natural language generation. In Proceedings of First North American ACL, Seattle, USA, May.

Ehud Reiter. 1994. Has a consensus NL generation architecture appeared, and is it psycholinguistically plausible? In Proceedings of the 7th International Workshop on Natural Language Generation, pages 163-170, Maine.

The XTAG-Group. 1999. A lexicalized Tree Adjoining Grammar for English. Technical Report http://ww. cis.upenn.edu/ ${ }^{x t a g /}$ tech-report/tech-report.html, The Institute for Research in Cognitive Science, University of Pennsylvania. 